\title{
Presença de sarcocystis cruzi em amostra fecal de cão, relato de caso
}

Dentre as possíveis doenças parasitárias que podem acometer os cães, estão as causadas por protozoários, como é o caso do Sarcocystis cruzi, agente etiológico da sarcocistose. Este parasito apresenta ciclo heteroxênico, necessitando além de um hospedeiro definitivo, um intermediário. $O$ cão se infecta ao ingerir carne crua ou vísceras contendo cistos viáveis. Alguns dos sinais clínicos são febre, anorexia, prostração, palidez das mucosas, corrimento nasal e ocular e dispneia. 0 exame coproparasitológico para pesquisa dos esporocistos é uma das formas de diagnóstico. O objetivo deste relato é descrever o caso de um cão com esta parasitose, evidenciando o diagnóstico e o tratamento do mesmo.

Palavras-chave: Sarcocistose; Diagnóstico; Cães

\section{Presence of sarcocystis cruzi in a fecal sample of a dog, case report}

Among the possible parasitic diseases that can affect dogs are those caused by protozoa, such as Sarcocystis cruzi, the etiological agent of sarcocystosis. This parasite presents a heteroxenic cycle, requiring, besides a definitive host, an intermediate. The dog is infected by ingesting raw meat or viscera containing viable cysts. Some of the clinical signs are fever, anorexia, prostration, mucosal pallor, nasal and ocular discharge and dyspnea. Coproparasitological examination for sporocysts is one of the diagnostic methods. The objective of this report is to describe the case of a dog with this parasitosis, evidencing the diagnosis and treatment of the same.

Keywords: Sarcocystosis; Diagnosis; Dogs.

Alexsander Ferraz (iD)

Universidade Federal de Pelotas, Brasil http://lattes.cnpq.br/5047550122468432 http://orcid.org/0000-0002-0424-6249 xanderferraz@yahoo.com.br

Bruna dos Santos Pires (iD

Universidade Federal de Pelotas, Brasil http://lattes.cnpq.br/2019874644735527 http://orcid.org/0000-0002-3755-6243 bruspires@gmail.com

Eugênia Tavares Barwaldt (D)

Universidade Federal de Pelotas, Brasil http://lattes.cnpq.br/4970989236096941 http://orcid.org/0000-0002-4902-1203 tbeugenia@gmail.com

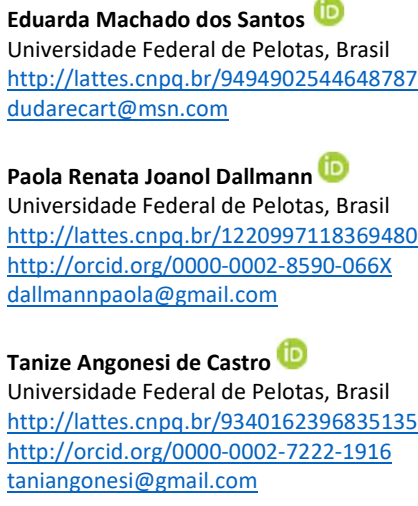

Márcia de Oliveira Nobre (ID

Universidade Federal de Pelotas, Brasil http://lattes.cnpq.br/3741542973280238 http://orcid.org/0000-0003-3284-9167 marciaonobre@gmail.com

Leandro Quintana Nizoli (in)

Universidade Federal de Pelotas, Brasil http://lattes.cnpq.br/3556266527587195 http://orcid.org/0000-0002-0767-4097 leandro.nizoli@gmail.com

\section{Referencing this:}

FERRAZ, A.; PIRES, B. S.; BARWALDT, E. T.; SANTOS, E. M.; DALLMANN, P. R. J.; CASTRO, T. A.; NOBRE, M. O.; NIZOLI, L. Q.. Presença de sarcocystis cruzi em amostra fecal de cão, relato de caso. Scire Salutis, v.10, n.1, p.14-17, 2020. DOI: http://doi.org/10.6008/CBPC22369600.2020 .001 .0003 


\section{INTRODUÇÃO}

Os coccídeos são responsáveis por diversas enfermidades, tanto em animais como em humanos. Dentre estas patologias, algumas são causadas pelo filo Apicomplexa, como é o caso da família Sarcocystidae que inclui o gênero Sarcocystis, agente etiológico da sarcocistose (CORLISS, 1994). Os parasitos deste gênero, possuem ciclo heteroxênico, ou seja, necessitam de um hospedeiro intermediário (HI) para a fase assexuada de seu ciclo e um hospedeiro definitivo (HD) para a fase sexuada (TENTER, 1995). Por esta característica, estão associadas ao ciclo presa-predador (LOPES, 2004).

Este protozoário tem como hospedeiros definitivos, animais carnívoros como o cão, o gato ou seres humanos e possuem vários hospedeiros intermediários como as aves, os répteis, pequenos roedores, herbívoros e suínos (CARLTON et al., 1995). Os HI são infectados através da ingestão de oocistos liberados nas fezes dos HD e estes por sua vez, infectam-se a partir da ingestão de cistos, contidos em vísceras ou carne crua do HI (DUBEY et al., 2006).

Dentre os sinais clínicos que podem ser observados, destacam-se febre, anorexia, diarreia, palidez das mucosas, corrimento nasal e ocular, dispneia, salivação e prostração, em fêmeas, ainda pode ocorrer abortos, retenção de placenta e nascimento de animais fracos (LOPES, 2004). Sarcocystis cruzi é a principal espécie que acomete os cães, tendo o bovino como HI (RUAS et al., 2001). S. levinei, S. capracanis, S. tenella e S. miescheriana, cujos HI são, respectivamente, Búfalos, caprinos, ovinos e suínos, também podem infectar os cães (ODENING, 1998). Com base em análise de tecidos em animais de abatedouro, Van Knapen et al. (1987), verificaram que existe uma elevada prevalência de bovinos abatidos em todo mundo infectados com cistos deste parasito.

Nos cães, a prevalência varia conforme o país, período e a metodologia utilizada no estudo (FONTANARROSA et al., 2006; MARTÍNEZ-MORENO et al., 2007; SAGER et al., 2006). Uma das formas de diagnóstico é através do exame coproparasitológico, sendo possível, através deste, a observação dos esporocistos (FORTES, 2004). O objetivo deste trabalho foi relatar um caso de infecção por Sarcocystis cruzi em canino.

\section{RELATO}

Foi recebido no Laboratório de Doenças Parasitárias (Ladopar) da Faculdade de Veterinária (Favet) da Universidade Federal de Pelotas (UFPel), uma amostra fecal, procedente de um cão, macho, adulto e sem raça definida. 0 animal havia sido adotado há cerca de 10 dias pelo tutor, que relatou que o mesmo, apresentava-se magro e com sintomatologia gastrintestinal, como diarreia, com presença de sangue e episódios de vômito.

Devido a estas alterações, foi encaminhado material para realização de análise coproparasitológica, tendo como objetivo, a pesquisa de parasitos gastrintestinais e a partir do diagnóstico, proceder o tratamento ideal. As técnicas utilizadas para diagnóstico foram: Willis-Mollay (1921), que utiliza o princípio da flutuação em solução hipersaturada, para pesquisa de ovos leves de helmintos e oocistos de protozoários 
e Faust et al. (1938), que consiste na centrifugo-flutuação em solução de sulfato de zinco a 33\%, utilizada para pesquisa de cistos de Giardia sp. e oocistos

\section{RESULTADOS}

Na técnica de Willis-Mollay (1921), foram observados ovos de Ancylostoma spp., e Trichuris sp. (Figura 1). Na técnica de Faust et al. (1938), foi observado a presença de esporocistos de S. cruzi (Figura 2). A partir do diagnóstico, foi instituído tratamento com clindamicina $15 \mathrm{mg} / \mathrm{kg}, 12 \times 12$ horas, via oral, durante 21 dias, visando a eliminação do Sarcocystis. Para combater os helmintos Ancylostoma spp e Trichuris sp. foi utilizado vermífugo de amplo espectro, a base de Pamoato de Pirantel, Praziquantel e Febantel, duas doses, com intervalo de 14 dias.

Após o término do tratamento, foi realizado novo exame coproparasitológico, utilizando as mesmas técnicas anteriores, onde constatou-se que o paciente estava negativo para todos os gêneros de parasitos, evidenciando a eficácia do tratamento. A prevalência deste protozoário na população canina está relacionada aos hábitos alimentares desses animais. Os cães que se alimentam de carne crua, através de pequenos mamíferos e pássaros ou pelo fornecimento de carnes e vísceras cruas ou malcozidas, contendo cistos musculares viáveis, apresentam um maior risco de infecção por este protozoário (LABRUNA et al., 2006).

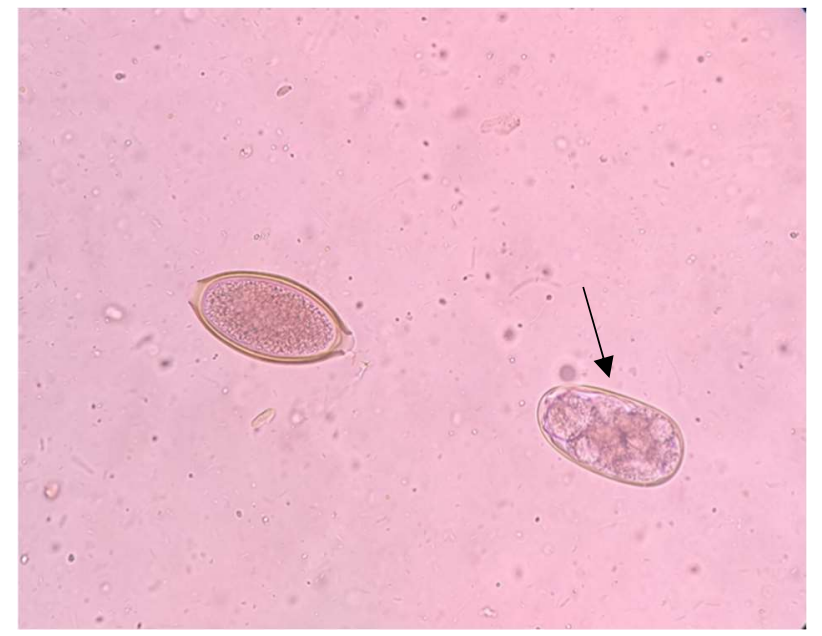

Figura 1: Ovos de Ancylostoma spp. (seta) e Trichuris sp. observados na técnica de Willis-Mollay, aumento de $100 x$

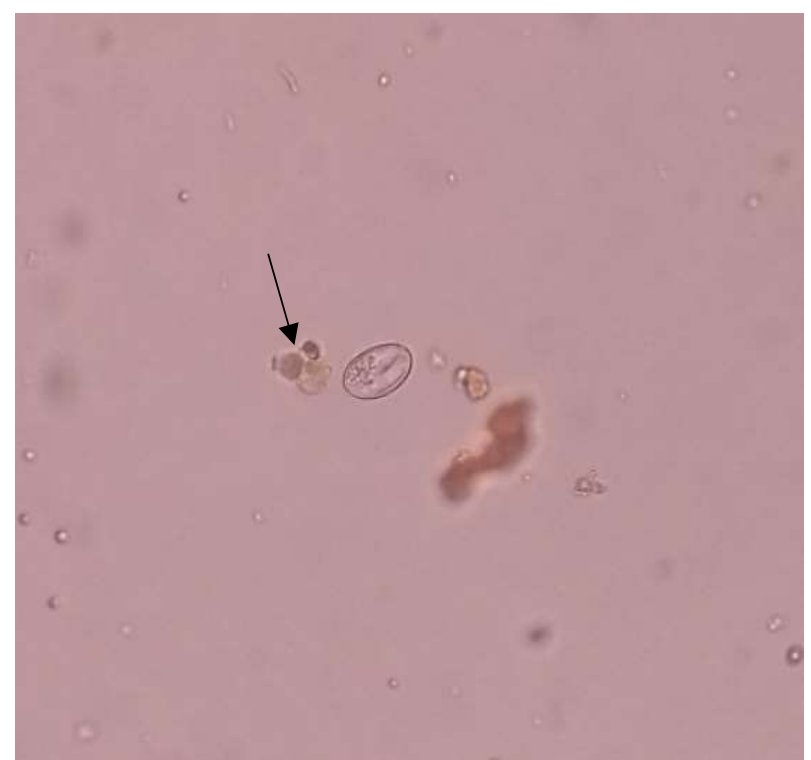

Figura 2: Esporocisto de Sarcocystis cruzi (seta), observado na técnica de Faust, aumento de 400x.

Alguns estudos evidenciam a presença de S. cruzi em amostras fecais de cães, como o de Alves et al. (2005), que analisando a presença de enteroparasitos em cães domiciliados no município de Goiânia, encontraram uma prevalência de 0,26\% para este gênero, assim como Leal et al. (2016) que em 221 amostras de fezes de cães domiciliados atendidos em serviço de saúde animal no Rio de Janeiro, observaram que 0,45\% foram positivas para este parasito.

A baixa frequência de infecção em cães domiciliados pode ser explicada pelo hábito alimentares destes animais, que na maioria das vezes alimenta-se de ração, enquanto os de vida livre e os que vivem em áreas rurais, tem mais acesso a carne crua do hospedeiro intermediário, contendo cistos (LOPES, 2004). 


\section{CONSIDERAÇÕES FINAIS}

Levando em conta o ciclo do parasito e sua forma de transmissão, algumas medidas são necessárias para que seu controle seja possível, como evitar que o cão ingira carne crua ou malcozida do hospedeiro intermediário, visto que está pode conter cistos infectantes. Desta forma, não ocorrerá infecção do HD, que por sua vez, não eliminará esporocistos nas fezes, evitando a contaminação do ambiente e consequentemente dos $\mathrm{HI}$, impedindo o desenvolvimento de ciclo.

\section{REFERÊNCIAS}

ALVES, O. F.; GOMES, A. G.; SILVA, A. C.. Ocorrência de enteroparasitos em cães do município de Goiânia, Goiás: comparação de técnicas de diagnóstico. Ciência Animal Brasileira, v.6, n.2, p.127-133, 2005.

CORLISS, J. O.. An interim utilitarian ('user-friendly') hierarchical classification and characterization of the protists. Acta Protozoologica, v.33, p.1-1, 1994.

DUBEY, J. P.; LINDSAY, D. S.. A review of Neospora caninum and neosporosis. Veterinary Parasitology, v.67, p.1-59, 1996.

FAUST, E. C.; D'ANTONI, J. S.; ODOM, V.. A critical study of clinical laboratory technics for the diagnosis of protozoan cysts and helminth eggs in feces I. Preliminary communication. American Journal of Tropical Medicine, v.18, p.169-183, 1938.

FONTANARROSA, M. F.; VEZZANI, D.; BASABE, J.; EIRAS, D. F.. $\mathrm{Na}$ epidemiological study of gastrointestinal parasites of dogs from Southern Greater Buenos Aires (Argentina): Age, gender, breed, mexe infections, and seasonal and spatial patterns. Veterinary Parasitology, v.136, n.3-4, p.283-95, 2006.

FORTES, E.. Parasitologia Veterinária. 4 ed. São Paulo: Cone, 2004. 137p.

LABRUNA, M. B.; PENA, H. F. J.; SOUZA, S. L. P.; PINTER, A.; SILVA, J. C. R.; RAGOZO, A. M. A.; CAMARGO, L. M. A.; GENNARI, S. M.. Prevalência de endoparasitas em cães da área urbana do município de Monte Negro, Rondônia. Arquivos do Instituto Biológico, v.73, n.2, p.183-193, 2006.

LEAL, P. D. S.; MORAES, M. I. M. R.; BARBOSA, L. L. O.; FIGUEIREDO, L. P.; SILVA, S. L.; LOPES, C. W. G.. Parasitos gastrintestinais em cães domiciliados atendidos em serviço de saúde animal no Rio de Janeiro, Brasil. Revista Brasileira de Medicina Veterinária, v.37, n.1, p.37-44, 2015.

LOPES, C. W. G.. O gênero Sarcocystis (Lankester, 1882) (Apicomplexa:Sarcocystidae), uma questão a ser reavaliada no Brasil. Revista Brasileira de Medicina Veterinária, v.13, n.1, p.14-16, 2004.

ODENING, K.. The present state of species-systematics in Sarcocystis Lankester, 1882 (Protista, Sporozoa, Coccidia). Systematic Parasitology, v.41, n.3, p.209-233, 1998.

MARTÍNEZ-MORENO, F. J.; HERNÁNDEZ, S.; LÓPEZ-COBOS, E.; BECERRA, C.; ACOSTA, I.; MARTÍNEZ-MORENO, A. Estimation of canine intestinal parasites in Córdoba (Spain) and their risk to public health. Veterinary Parasitology, v.143, n.1, p.7-13, 2007.

RUAS, J. L.; CUNHA, C. W.; SILVA S. S.. Prevalência de Sarcocystis spp. (Lankester, 1882) em bovinos clinicamente sadios, da região do Rio Grande do Sul, Brasil. Revista Brasileira de Agrociências, v.7, p.227-230, 2001.

SAGER, H.; MORET, C. S.; MÜLLER, N.; STAUBLI, D.; ESPOSITO, M.; SCHARES, G.; HÄSSIG, M.; STÄRK, K.; GOTTSTEIN, B.. Incidence of Neospora caninum and other intestinal protozoan parasites in populations of Swiss dogs. Veterinary Parasitology, v.139, n.1-3, p.84-92, 2006.

TENTER, A. M.. Current research on Sarcocystis species of domestic animals. International Journal of Parasitology, v.25, p.1311-1330, 1995.

VAN KNAPEN, F.; BOUWMANN, D.; GREVE, E.. Study on the incidence of Sarcocystis spp.in dutch cattle using various methods. Tijdschr Diergeneeskd, v.112, p.1095-1100, 1987.

WILLIS-MOLLAY, H. H.. A simple levitation method for the detection of hookworm ova. The Medical Journal Australia. v.2, n.18, p.375-376, 1921.

A CBPC - Companhia Brasileira de Produção Científica (CNPJ: 11.221.422/0001-03) detém os direitos materiais desta publicação. Os direitos referem-se à publicação do trabalho em qualquer parte do mundo, incluindo os direitos às renovações, expansões e disseminações da contribuição, bem como outros direitos subsidiários. Todos os trabalhos publicados eletronicamente poderão posteriormente ser publicados em coletâneas impressas sob coordenação da Sustenere Publishing, da Companhia Brasileira de Produção Científica e seus parceiros autorizados. Os (as) autores (as) preservam os direitos autorais, mas não têm permissão para a publicação da contribuição em outro meio, impresso ou digital, em português ou em tradução. 